https://doi.org/10.22319/rmcp.v12i2.5519

Nota de investigación

\title{
Caracterización de la curva de lactancia y calidad de la leche en ovejas Santa Cruz (Ovis aries)
}

Ingrid Merchant $^{\mathrm{a}}$

Agustín Orihuela ${ }^{\text {a* }}$

Reyes Vázquez ${ }^{\text {a }}$

Virginio Aguirre ${ }^{a}$

${ }^{a}$ Universidad Autónoma del Estado de Morelos. Facultad de Ciencias Agropecuarias. Avenida Universidad 1001 Colonia Chamilpa, Cuernavaca Morelos 62210, México.

* Autor de correspondencia: aorihuela@uaem.mx

\section{Resumen:}

En las ovejas de pelo existe muy poca información respecto a las características de la producción y calidad de la leche a lo largo de la lactancia, pese a sus implicaciones tanto en la sobrevivencia y mantenimiento de los corderos, como en la alimentación humana. Con el propósito de caracterizar la curva de lactancia y la calidad de la leche en ovejas Santa Cruz, se utilizaron 18 ovejas multíparas que parieron en un lapso de cuatro días. La producción láctea se registró cada $72 \mathrm{~h}$ en el periodo comprendido de 6 a 60 días postparto (dpp), mientras que la calidad de la leche se determinó una vez por semana de una muestra de la producción total del día. La producción de leche 6 dpp fue de $1.95 \mathrm{~L}$, alcanzando un máximo de $2.31 \mathrm{~L}$, 12 dpp y disminuyendo hasta 1.01, a los $57 \mathrm{dpp}$. El porcentaje de sólidos totales fue $18 \%$, incrementando hasta $20.5 \%$ en la octava semana. El porcentaje de grasa durante la segunda semana fue $8 \%$ incrementándose hasta $9.8 \%$ al final del periodo evaluado, mientras que los porcentajes de proteína y lactosa se mantuvieron relativamente uniformes durante todo el periodo experimental en niveles entre 4.86 y $5.18 \%$ para proteína, y 4.68 a $4.74 \%$ para lactosa. Se concluye que la producción de leche en ovejas Santa Cruz, alcanza su máximo alrededor de la segunda semana de lactancia, disminuyendo a partir de ese momento de 
manera constante y paulatina, incrementando los porcentajes de sólidos totales y grasa a través del tiempo, mientras que los de proteína y lactosa se mantienen constantes.

Palabras clave: Proteína, Lactosa, Grasa, Sólidos totales, Producción de leche, Ovinos.

Recibido: 26/09/2019

Aceptado: 23/07/2020

En las zonas tropicales, la ovinocultura está enfocada principalmente hacia la producción de carne, sobresaliendo las razas de pelo debido a su capacidad de adaptación a temperaturas elevadas, resistencia a parásitos y prolificidad alta ${ }^{(1)}$. En este tipo climas, el sistema productivo más común es el extensivo, donde el número de corderos criados tiene el mayor valor para la producción, representando la principal fuente de ingresos para el productor $^{(2)}$.

La producción de leche en estas razas, tiene un valor fundamental para la supervivencia, crecimiento y peso al destete de los $\operatorname{corderos}^{(3,4)}$, y en ocasiones llega a ser una fuente alternativa de proteína para el consumo humano. Pese a lo anterior, la información respecto a la producción de leche en ovinos de pelo se limita a pocos estudios en razas como la Santa Cruz, Blackbelly, Katahdin ${ }^{(5-7)}$ y algunas otras ovejas del oeste de África ${ }^{(8)}$, mientras que la composición nutritiva de la leche, sólo se ha caracterizado en Blackbelly X Katahdin ${ }^{(9,10)}$, lo que refleja la poca información respecto a la producción y calidad de la leche durante la lactancia en ovinos de pelo, pese a su popularidad en la región tropical de Norte y Centro América ${ }^{(11,12)}$.

El consumo diario de leche es el factor más importante en la tasa de crecimiento de los corderos; por lo que la supervivencia, potencial de crecimiento y peso al destete de las crías, dependerá de la producción de leche a través de la lactancia de la madre ${ }^{(3,4)}$. Sin embargo, existe una gran variación en los genotipos de las razas tropicales, por lo que la estimación de la producción láctea a lo largo de la lactancia, así como la variación en su composición es información indispensable para establecer estrategias de manejo para ovejas y corderos. Por lo anterior, el objetivo del presente estudio fue caracterizar la producción y calidad de la leche en ovejas Santa Cruz, desde el parto hasta el destete.

El estudio se realizó en el campo experimental de la Facultad de Ciencias Agropecuarias de la Universidad Autónoma del Estado de Morelos, ubicado a $18^{\circ} 56^{\prime} \mathrm{N}$ y $99^{\circ} 13^{\prime} \mathrm{O}$, a una altitud de 2,160 msnm. La temperatura anual promedio es de $20^{\circ} \mathrm{C}$, con una precipitación media anual de $1,243 \mathrm{~mm}$. 
Se utilizaron 18 ovejas multíparas Santa Cruz con edad y peso promedio de 2.0 años y 51.22 $\pm 2.36 \mathrm{~kg}$, respectivamente, que parieron dentro de un rango de cuatro días. De las ovejas consideradas, 12 parieron mellizos, 2 tuvieron partos triples y 4 partos sencillos. Durante la gestación, las ovejas se mantuvieron en un solo grupo, pastoreando de 0800 a 1400 h en una pradera establecida con pasto estrella africana (Cynodon nlemfluensis). El resto del día permanecían estabuladas recibiendo una suplementación de $300 \mathrm{~g}$ de concentrado comercial. Este concentrado aportó $16 \%$ de PC y $2.79 \mathrm{Mcal} / \mathrm{kg}$ de EM, mientras que el forraje (alfalfa + sorgo) proporcionaron $7.6 \%$ de PC y $1.78 \mathrm{Mcal} / \mathrm{kg}$ de EM. Considerando que la dieta incluyó $50 \%$ de concentrado y $50 \%$ de forraje, ésta cubría los requerimientos nutricionales de una oveja de $60 \mathrm{~kg}$, con dos crías, produciendo entre 0.79 y $1.48 \mathrm{~kg}$ de leche por día ${ }^{(13)}$. Durante la lactancia, las ovejas se alimentaron con $1 \mathrm{~kg}$ del mismo alimento concentrado comercial, agua y una mezcla de forraje (30\% alfalfa y $70 \%$ paja de sorgo) a libre acceso. Los primeros 5 días posparto (dpp) cada oveja con sus crías, se alojó en corrales individuales de 2 x $2 \mathrm{~m}$, techados, con piso de cemento, comedero y bebedero individual. A partir del sexto dpp hasta el momento del destete $(60 \mathrm{dpp})$ todas las hembras con sus crías se mantuvieron en un solo grupo dentro de un corral ( $4 \mathrm{~m}^{2} /$ oveja), techado, piso de cemento, comedero y bebedero compartido. El corral contaba con un área de suplementación para las crías (creep feeding) donde se les ofreció alimento concentrado comercial al $18 \%$ de PC a partir de los $14 \mathrm{dpp}$, a libre acceso.

La producción láctea de las ovejas se midió a partir del 6to dpp, cada tres días, mediante el "método de la oxitocina", el cual consiste en separar a los corderos de las hembras y aplicar 5 UI de oxitocina sintética por vía intravenosa (Oxitopisa; Pisa; Hidalgo, México) y ordeñar la ubre por completo de forma manual. Transcurridas $4 \mathrm{~h}$ de la aplicación de la hormona, se aplicó una segunda dosis y se ordeñó nuevamente, registrando el volumen de leche obtenida. Este valor se multiplicó por 6, para estimar la producción diaria ${ }^{(14)}$.

La calidad de la leche se determinó una vez por semana a partir de una muestra de $50 \mathrm{ml}$ de leche recolectada del total de la ordeña diaria. El análisis de laboratorio (Alimenlab; Jalisco, México) determinó la concentración de proteína, grasa, lactosa y sólidos totales en la leche mediante el método de espectrofotometría infrarroja ${ }^{(15)}$.

Para el análisis estadístico de la producción y calidad de leche se realizó una regresión polinómica para obtener la ecuación, el valor de $\mathrm{R}^{2}$ y su probabilidad mediante el procedimiento PROC REG ${ }^{(16)}$.

La producción de leche al día 6 postparto fue de $1.95 \mathrm{~L}$, incrementándose hasta alcanzar un máximo alrededor de $12 \mathrm{dpp}$ con $2.31 \mathrm{~L}$, disminuyendo posteriormente hasta $1.01 \mathrm{~L}$ a los 57 dpp. 
Al dividir la curva de lactancia en cinco períodos de 9 días, se observó que en los dos primeros periodos (equivalentes a $18 \mathrm{dpp}$ ) no existe diferencia entre ellos $(P>0.05)$. Sin embargo, en los siguientes periodos se observa una disminución $(P<0.05)$ gradual; $15 \%$ en el 3er periodo, $26 \%$ en el 4to periodo y del $39 \%$ en el 5 to periodo con respecto a los 2 primeros. El peso promedio de las ovejas fue similar a lo largo de la lactancia $(P>0.05)$, registrando $52.24 \pm 1.19$ y $52.00 \pm 1.63 \mathrm{~kg}$ al parto y destete, respectivamente.

En el análisis de regresión correspondiente (Figura 1), se observa una relación inversamente proporcional en el tiempo, ajustada a una línea de tendencia polinómica con $\mathrm{r}^{2}=0.88 ; \mathrm{y}=-$ $0.0003 x^{2}-0.0513 x+2.1079(P=0.0001)$.

Figura 1: Curva de lactancia de ovejas Santa Cruz durante los primeros 60 Días post parto (media $\pm \mathrm{EE})(P \leq 0.001)$. Regresión polinómica

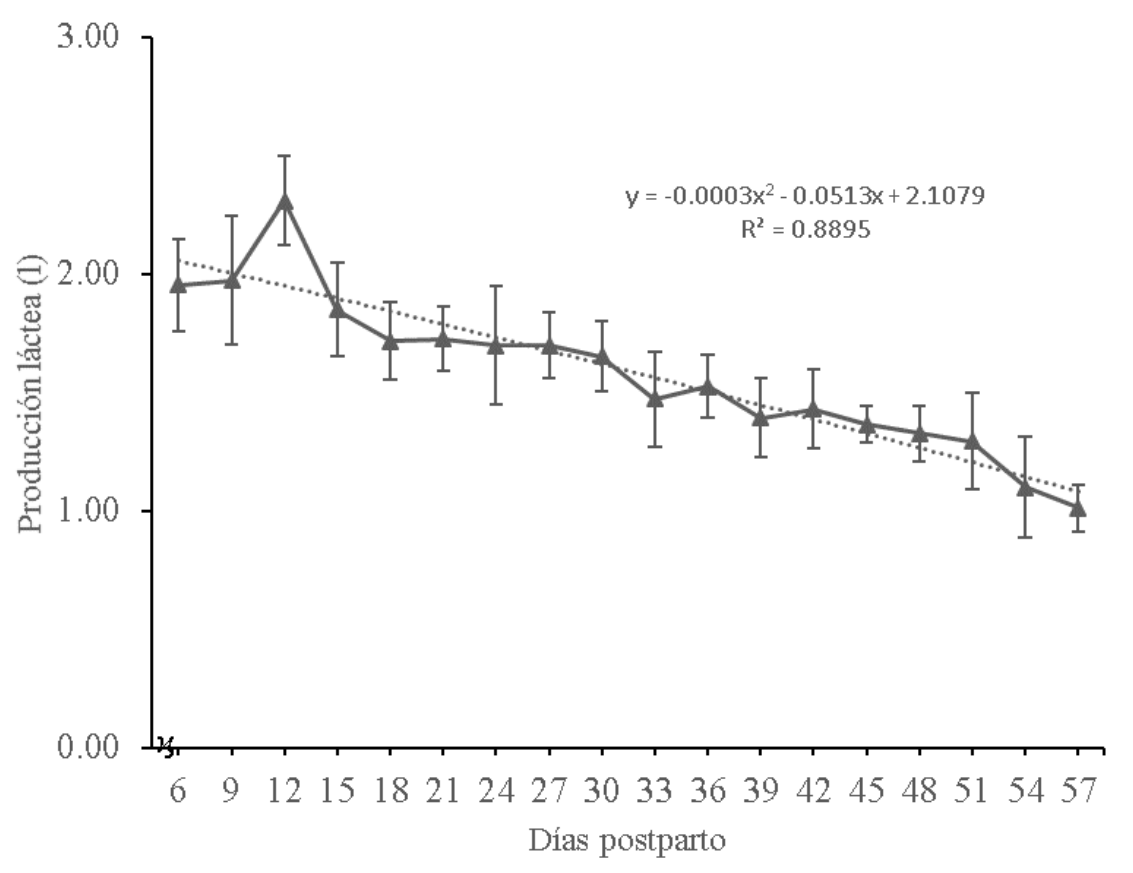

Durante la segunda semana de lactancia (Figura 2), el porcentaje de sólidos totales en la leche fue de $18 \%$, incrementando hasta el $20.5 \%$ en la octava semana. En el análisis de regresión correspondiente se observa una relación proporcional en el tiempo, con $\mathrm{r}^{2}=0.92$; $\mathrm{y}=-$ $0.0993 x^{2}+1.1487 x+17.276(P=0.01)$. 
Figura 2: Composición de la leche de ovejas de pelo de la raza Santa Cruz durante los primeros 60 días post parto (media $\pm \mathrm{EE})(P \leq 0.001)$

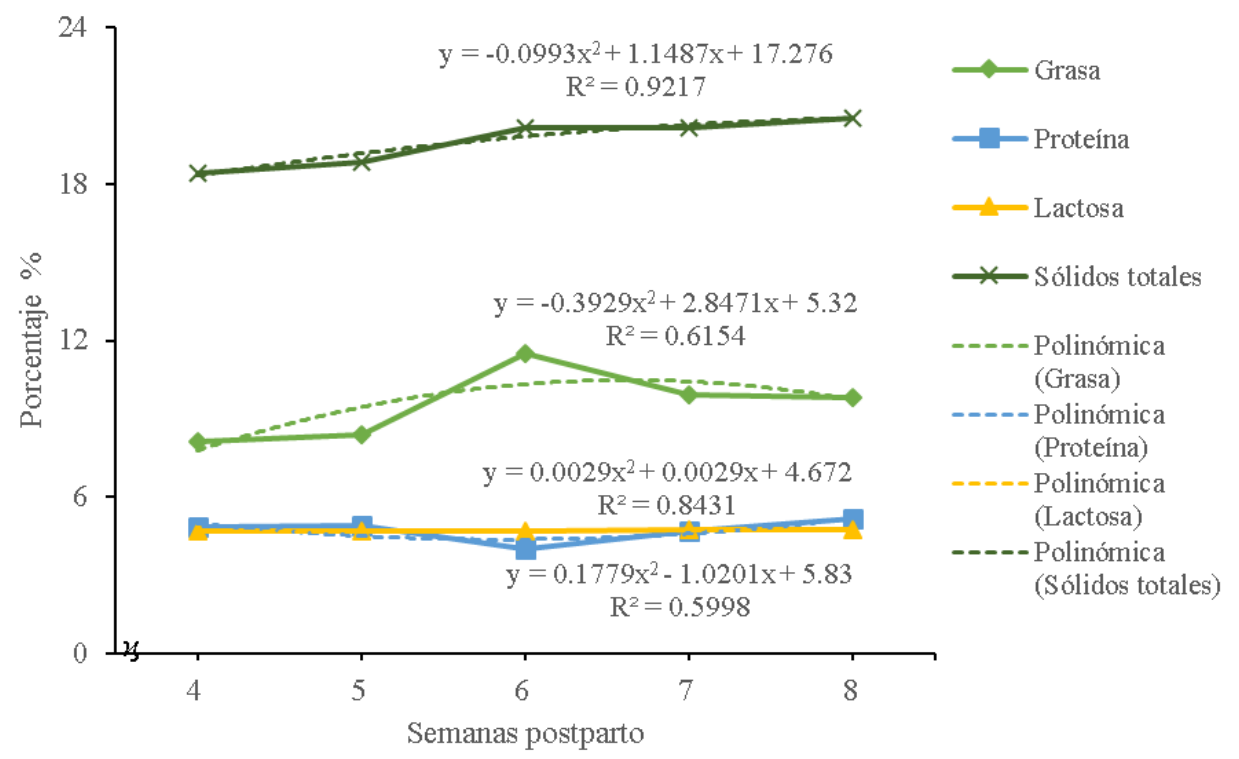

El porcentaje de grasa en la leche durante la segunda semana fue de $8 \%$ incrementándose hasta $9.8 \%$ al final de la lactancia. El análisis de regresión confirma esta tendencia con una $\mathrm{r}^{2}=0.61 ; \mathrm{y}=-0.3929 \mathrm{x}^{2}+2.8471 \mathrm{x}+5.32(P=0.23)$.

Los porcentajes de proteína y lactosa durante la segunda semana fueron de 4.86 y $4.68 \%$ para proteína y lactosa, respectivamente. Al final de la lactancia los valores correspondientes fueron de: $5.18 \%$ y $4.75 \%$. El análisis de regresión muestra muy poca fluctuación a lo largo de la lactancia para ambas variables. En el caso de proteína, se obtuvo una $\mathrm{r}^{2}=0.84$; $\mathrm{y}=0.0029 \mathrm{x}^{2}+0.0029 \mathrm{x}+4.672(P=0.90)$, mientras que para lactosa la $\mathrm{r}^{2}=0.60 ; \mathrm{y}=0.1779 \mathrm{x}^{2}-$ $1.0201 x+5.83(P=0.05)$.

La producción láctea en ovejas Santa Cruz muestra un máximo a los 12 dpp, sin apreciarse un claro pico de lactancia para posteriormente disminuir paulatina y constante. En general, la máxima secreción de leche en las diferentes razas ovinas se produce entre la segunda y cuarta semanas de lactancia ${ }^{(17)}$. En el caso de la Santa Cruz, este periodo de máxima producción coincide con hallazgos previos en ovejas Katahdin ${ }^{(7)}$, así como los de ovejas Ile de France ${ }^{(18)}$ y otras razas ${ }^{(19)}$. Sin embargo, la cantidad de leche producida durante este mismo periodo en estas razas cárnicas fue mayor en Santa Cruz (2.33 L/día) en comparación con las razas antes mencionadas $(1.38,0.50$ y 0.43 , respectivamente), resaltando la alta producción de leche en las razas de pelo ${ }^{(20)}$ en comparación con la información de razas cárnicas de origen europeo. En el único estudio encontrado por los autores que caracteriza la producción de leche a lo largo de la lactancia en ovejas Santa $\mathrm{Cruz}^{(7)}$, tampoco les fue posible identificar un pico máximo de producción láctea. Situación que podría deberse al alto contenido 
energético que se proporcionó en la dieta de estos trabajos ${ }^{(21-23)}$, considerando que se ofreció el mismo alimento concentrado, variando únicamente el forraje (alfalfa+paja de sorgo vs ensilado de maíz), ofrecido en ambos casos a libre demanda.

En la composición química de la leche de las ovejas Santa Cruz, los porcentajes de sólidos totales y grasa incrementaron al final de la lactancia. Lo anterior resulta lógico debido a que en general, al disminuir la producción láctea, se concentran los sólidos totales y la grasa, situación observada en otras razas ${ }^{(24)}$, incluyendo las especializadas en la producción de leche ${ }^{(25-26)}$, lo que a su vez, se asocia con leches de menor calidad ${ }^{(27)}$.

Por otra parte, llama la atención que, en el presente experimento, los porcentajes de proteína y lactosa se mantuvieron constantes a través del tiempo, lo que, dada la disminución total de leche conforme avanza la lactación, significarían un incremento de estas sustancias conforme avanza el tiempo. Esto podría ser una ventaja importante en las características nutricionales de la leche de esta raza. Sin embargo, se recomienda realizar mayor investigación con el fin de confirmar este hallazgo.

La mayor concentración de proteína y lactosa en la leche podría deberse a que las ovejas tropicales viven en condiciones húmedas, lo que reduce los requerimientos de agua de las crías a través de la leche. Una situación inversa se ha encontrado en otras especies que habitan climas desérticos, donde la cantidad de agua en la leche se incrementa ${ }^{(27)}$. Por otra parte, las razas tropicales son más ligeras que las europeas, por lo que menores requerimientos de mantenimiento, podrían permitir destinar mayor cantidad de nutrientes para una mejor calidad de leche ${ }^{(19)}$.

Se concluye que la producción de leche en ovejas Santa Cruz, alcanza su máximo alrededor de la segunda semana de lactancia, disminuyendo a partir de ese momento, de manera constante y paulatina, incrementando los porcentajes de sólidos totales y grasa a través del tiempo, mientras que los de proteína y lactosa se mantienen constantes.

\section{Conflicto de intereses}

Los autores declaran no tener conflicto de intereses en relación con el presente trabajo.

\section{Agradecimientos}

Se agradece al CONACyT por la beca otorgada para la realización de los estudios de doctorado en Ciencias Agropecuarias y Desarrollo Rural a la MC Ingrid Merchant Fuentes con número de becario: 410994. 


\section{Literatura citada:}

1. Quintanilla-Medina JJ, González-Reyna A, Hernández-Meléndez J, Limas-Martínez AG, Carreón-Pérez A, Martínez-González JC. Producción de ovinos de pelo bajo condiciones de pastoreo en el noreste de México. Rev Invest Vet Peru 2018;(29):544551.

2. FAOSTAT. Estadísticas | FAO | Organización de las Naciones Unidas para la Alimentación y la Agricultura. División de las Estadísticas de la Organización de las Naciones Unidas para la Alimentación (FAO). 2013.

3. Banchero GE, Quintans G, Milton JT, Lindsay DR. Alimentación estratégica para mejorar la lactogénesis de la oveja al parto. Seminario de actualización técnica: Reproducción Ovina. INIA 2005;(33 Tacuarembó):127-136.

4. Millanao I, Herdener N, Parada D, Sepúlveda N. Producción de leche , curvas de lactancia y crecimiento de sus corderos, en dos razas de ovejas en la región de la Araucanía Chile. Sitio Argentino Prod Anim 2007;(APPA-ALPA):1-4.

5. Godfrey RW, Gray ML, Collins JR. Lamb growth and milk production of hair and wool sheep in a semi-arid tropical environment. Small Ruminant Res 1997;(24):77-83.

6. Peniche-Gonzalez I, Sarmiento-Franco L, Santos-Ricalde R. Estimation of milk production in hair ewes by two methods of measurement. Rev MVZ Córdoba 2015;(20):4629-4635.

7. Burgos-González C, Huerta-Aparicio M, Aguirre V, Vazquez R, Orihuela A, Pedernera M. Short communication: Milk production and lamb development in Saint Croix and Katahdin hair sheep breeds (Ovis aries). Trop Anim Health Prod 2017;(3):683-687.

8. Ünal N, Akçapinar H, Atasoy F, Yakan A, Uğurlu M. Milk yield and milking traits measured with different methods in Bafra sheep. Rev Med Vet (Toulouse). 2008;(159):494-501.

9. Araujo RC, Pires AV., Susin I, Mendes CQ, Rodrigues GH, Packer IU, et al. Milk yield, milk composition, eating behavior, and lamb performance of ewes fed diets containing soybean hulls replacing coastcross (Cynodon species). J Anim Sci 2008;(86):35113521.

10. Peniche-Gonzalez I, Sarmiento-Franco LA, Santos-Ricalde RH. Utilization of Mucuna pruriens whole pods to feed lactating hair ewes. Trop Anim Health Prod 2018;(7):14551461.

11. Notter DR. Effects of ewe age and season of lambing on prolificacy in US Targhee, Suffolk, and Polypay sheep. Small Ruminant Res 2000;(38):1-7. 
12. Sánchez-Dávila F, Bernal H, Colín J, Olivares E, del Bosque AS, Ledezma R, et al. Environmental factors and interval from the introduction of rams to estrus in postpartum Saint Croix sheep. Trop Anim Health Prod 2011;(43):887-891.

13. Nutrient requirements of small ruminants; Sheep, goats, cervids, and new world camelids. Animal Nutrition Series, National Research Council of the National Academies. 2007.

14. Velasco S, Cañeque V, Díaz MT, Pérez C, Lauzurica S, Huidobro F. Producción lechera y composición lipídica de la leche de ovejas Talaveranas durante el período de lactancia. Investig Agrar Prod y Sanid Anim 2001;(16):182-192.

15. ISO. Determination of milkfat, protein and lactose content. 1999.

16. SAS. Proceedings of the Twenty Eighth Annual SAS Users Group International Conference. SAS Instit. Cary, NC; 2003.

17. Church D. Alimentos y alimentación del ganado. 2da ed. Montevideo, Uruguay; 1989.

18. Lopes-Zeola BNM, da Silva-Sobrinho GA, Hatsumura CT, Borghi TH, Viegas CR, Barbosa JC. Production, composition and processing of milk from ewes fed soybean seeds. Rev Bras Zootec 2015;(44):146-154.

19. De Souza-Emediato RM, Ramos-De Siqueira E, De Melo-Stradiotto M, Maestá SA, Gonçalves HC. Desempenho de ovelhas da raça Bergamácia alimentadas com dieta contendo gordura protegida. Rev Bras Zootec 2009;(38):1812-1818.

20. Forcada F, Thos J, Lopez M, Sierra I. Producción de leche de la raza Rasa Aragonesa en la fase de amamantamiento del cordero. Actas IX Jornadas SEOC 1984:161-164.

21. Resksupaphon J. Nutritional effects on mammary development and milk production: effects of prepartum protein supplements [Master thesis]. Universiy of New England; 1996.

22. Ripoll-Bosch R, Álvarez-Rodríguez J, Blasco I, Picazo R, Joy M. Producción de leche y crecimiento de corderos en la raza Ojinegra de Teruel. Inf Téc Económ Agrar 2012;(108):298-311.

23. Manterola H, Cerda D, Mira J, Pavlic A. Producción y composición de la leche en ovejas Merino precoz, Suffolk y Suffolk x Merino. Adv Prod Anim 2007;(32):59-70.

24. Pulina G, Nudda A. La produzione del latte. In: Pulina G, editor. L'alimentazione degli ovini da latte. Avenue Media, Bologna, Italy; 2001:9-31.

25. Bencini R, Pulina G. The quality of sheep milk: a review. Aust J Exp Agric 1997;(37):485-504. 
26. Pulina G, Macciotta N, Nudda A. Milk composition and feeding in the Italian dairy sheep. Ital J Anim Sci 2005;(4 Suppl. 1):5-14.

27. Hinde K, Milligan LA. Primate milk: Proximate mechanisms and ultimate perspectives. Evol Anthropol 2011;(20):9-23. 